\title{
Multilingualism and Public Goods Provision: An Experiment in Two Languages in Uganda*
}

\author{
Paul Clist ${ }^{\dagger} \&$ Arjan Verschoor ${ }^{\ddagger}$ \\ School of International Development, \\ University of East Anglia, \\ UK
}

18th August 2017

\begin{abstract}
Multilingualism is the global norm, but the implications of this for cooperation and public goods provision have not been studied before. We test whether the language in which a public goods game is played affects subjects' contributions amongst a bilingual population in eastern Uganda, finding that subjects contribute $30 \%$ more on average in the national language. This treatment effect is solely driven by those most associated with the local Gisu identity, for whom contributions are 43-74\% higher in the national language. This difference fits with Gisu culture's high value on self-reliance and low value on reciprocity and cooperation, due to a violent history of intense competition over land. Language is thus shown to affect cooperation, but only for individuals who both have different latent norms and for whom language activates these norms.
\end{abstract}

JEL Codes: C71, C92, O12

Keywords: Identity, Language, Cooperation

NB: The experimental script, data and code are available at https://paulclist. github.io

${ }^{*}$ Acknowledgements: We would like to thank Oriana Bandiera, Gary Charness, Bertil Tungodden, Daniel Zizzo, two anonymous referees, the editor, and participants at the 2014 Symposium on Economic Experiments in Developing Countries and the 2015 Network for Integrated Behavioural Science Conference for useful feedback and comments. Joshua Balungira and colleagues provided excellent research assistance. Any errors remain our own.

${ }^{\dagger}$ Corresponding author. paul.clist@uea.ac.uk

$\ddagger$ a.verschoor@uea.ac.uk 


\section{Introduction}

Most of the world's population is multilingual. Amongst the 200 or so countries in the world, there are 7,105 known living languages in current use (Lewis et al., 2013). Most readers of this article will live in countries with a clear and unambiguous 'national de facto language' which are populated by monolinguals. From a global perspective however, multilingualism is more common (Crystal, 1997). Romaine (2004, p.385) states that "Bilingualism and multilingualism are a normal and unremarkable necessity of everyday life for the majority of the world's population." Indeed, in developing countries, 'societal multilingualism', where multiple languages co-exist within a given society, is particularly prevalent. Romaine (2001, p.517) gives a telling picture of daily life:

"The average educated person in Hyderabad may use Telegu at home, Sanskrit at the temple, English at the university, Urdu in business, etc... In societies such as these, multilingualism is not an incidental feature of language use, but a central factor and an organising force in everyday life."

One would expect multilingualism to affect important economic interactions. People's willingness to cooperate and contribute to public goods, in particular, could be affected by the language in which they are appealed to. From psycholinguistics we know that the language spoken by a person frames the way in which she perceives and conceptualises the world: a multilingual person thus potentially has multiple frames at her disposal, each of which remains latent until appealed to by the language that activates it (Hong et al., 2000; Luna et al., 2008). If these frames of reference differ in terms of the value placed on cooperation and public spiritedness, then whether one or the other language is used for appeals to cooperate or contribute to a public good would matter for public goods provision and cooperation.

While the concepts of identity and frames have recently become established in economics, any link between these and different languages has largely been neglected. ${ }^{1}$ Our innovation is to treat language as a cue for one of a potential variety of frames, or identities, and investigate whether cooperation is affected accordingly. Specifically, we investigate multilingualism and multiple identities by conducting a linear one-shot public goods game

\footnotetext{
${ }^{1}$ More generally, languages are rarely studied by economists. Recent exceptions include a high profile statistical relationship between certain linguistic features and savings behaviour (Chen, 2013), the findings that in their weaker language bilinguals are less prone to cognitive biases (Keysar et al., 2012; Costa et al., 2014a) and more likely to make utilitarian moral judgements (Costa et al., 2014b), and the effect of language in strengthening identity (Aspachs-Bracons et al., 2008; Clots-Figueras and Masella, 2013). Within the experimental economics literature, language has been mentioned mainly as a factor to be controlled for (Roth et al., 1991) or seen as a proxy for ethnicity (Habyarimana et al., 2009), rather than worthy of study in its own right. Desmet et al. (2012, p.337) argue that linguistic distinctions are more objective than ethnic boundaries ("it is easier to judge whether two populations speak different languages than to decide whether two populations belong to different ethnicities, a more amorphous concept...") and thus more amenable to study. In our study, language is not a signal of the ethnicity of one's partner and so any treatment differences are due to a subject's own response to the language.
} 
(PGG) in two languages amongst a bilingual population. Both languages are used locally, with around $90 \%$ of the local population understanding both languages, and neither has a stronger claim to being the dominant language in public settings. In effect two lingua francas are found in the study area: Luganda and Lugisu. ${ }^{2}$ We thus study whether cooperation is affected by language use in a setting in which balanced bilingualism is the norm. ${ }^{3}$ In the area of eastern Uganda that we selected, the two languages are interchangeable for public purposes, so the language of the experimental script appeals to a particular identity without revealing the experiment's aims.

We find a strong treatment effect such that subjects in the Luganda treatment contribute $30 \%$ more to the public good. Moreover, this treatment difference is driven by subjects which are most associated with the local Gisu identity, but this can't be interpreted strictly according to ethnic groups. Whilst for others there is no treatment difference, those most associated with Gisu culture make different choices in the different languages, exposing different sets of norms and the ability of language to activate these. These results provide strong evidence that where those norms and languages are closely connected, individuals that have different latent norms may respond differently when appealed to in different languages. They may also help explain common findings of low provision of public goods in societies with high social divisions, described as "one of the most powerful hypotheses in political economy" (Banerjee et al., 2005, p.639).

The main contribution of our paper is in highlighting the role that language plays in mediating cooperation, by activating different norms. The diverse societies in today's world plagued by the absence of cooperation and willingness to contribute to public goods are, of course, usually multilingual societies. Our findings suggest to us that the role of language in these phenomena, through activating one of a variety of identities, including sectarian ones, is an important new area of study. In addition, we see our findings as contributing to two major literatures.

The first is research in economics on identities, recently summarised by Hoff and Stiglitz (2016). Akerlof and Kranton (2000) formally introduced 'a person's sense of self' into economic analysis, where identities impose costs of deviation from category norms. The presence and size of these dis-utilities are determined by the salience of a category in a given situation, and the strength of identification with a category. A body of experimental literature has since provided empirical support. ${ }^{4}$ This research makes use

\footnotetext{
${ }^{2}$ Luganda is a 'national language' and Lugisu is the language of the people (the Gisu) that historically dominate Bugisu, in eastern Uganda. The sub-county of Nakaloke, our study area, is found in Bugisu and ethnically highly diverse, resulting from large inflows of migrants in the early twentieth century.

${ }^{3}$ Balanced bilingualism is a term from linguistics that means equal proficiency in two languages.

${ }^{4}$ Benjamin et al. (2010) present a set of experiments in which priming ethnicity causes Asian-American subjects to make more patient decisions relative to a neutral prime, with the opposite effect for black subjects. Hoff and Pandey $(2006,2014)$ show that performance is affected by the salience of caste for lower-caste students in India, again using a neutral prime as a control. Benjamin et al. (2013) prime religious identity and find a range of different effects in the domains of cooperation, risk and reciprocity. Others compare subjects primed in different ways. LeBoeuf et al. (2010) present evidence in which the
} 
of priming, temporarily making a certain social category salient, which has a much longer history in psychology.

Experimental research on identity faces the challenge of priming appropriately. To bring an identity to the fore, what is a good priming technology? The method chosen is typically to administer a questionnaire on a pertinent topic shortly before some experimental decision. A common concern regarding the validity of this method is that experimenter demand effects may be driving the results (Zizzo, 2010). ${ }^{5}$ Sometimes it is possible to rely on subtle cultural cues. Hoff and Pandey $(2006,2014)$ obtain their treatment effect by calling out (for all subjects in a session to hear) the names of subjects (young boys), as well as the names of their father, paternal grandfather, village and caste. This is seemingly innocuous but highlights the boys' social identity.

The psychological literature provides a different priming technology: using different languages to prime different identities. This has rarely been adopted in the economics literature, with three exceptions (Lambarraa and Riener, 2015; Espinosa et al., 2015; Li, 2010) discussed in section 5. In contexts where multiple languages are commonly spoken, different aspects of subjects' identities are primed in a way that is more normal than filling in a questionnaire. ${ }^{6}$ If people have multiple identities, and they behave according to the one that is 'primed', then behaviour should be affected by the cues present in the social context. Our contribution to research on identities in economics is to treat language as a cue for one of a potential variety of identities, and investigate whether cooperation is affected accordingly.

The second major literature we contribute to is experimental research that uses public goods games (PGGs) to understand differences in cooperation and public goods provision between societies and groups within societies. ${ }^{7}$ Two strands of the previous literature are relevant here. First, natural (externally relevant) groups have been used to examine whether contributions are affected by information regarding whether one's partner shares a given group membership. The information has included village of residence (Etang et al., 2011), tribe (Bernhard et al., 2006), ethnicity (Habyarimana et al., 2009) and nationality (Finocchiaro Castro, 2008). Results show higher cooperation, trust and norm enforcement

salience of different social identities (e.g. student, socialite, family member) affect stated preferences for related goods. Cadsby et al. (2013) show that for women (but not men), priming a business identity relative to a family/gender identity increases the likelihood with which they choose a competition over a piece rate pay structure. Looking at cooperation, Chen et al. (2014) study prisoner's dilemma and minimum effort games where either a fragmenting (ethnic) or common (university) identity is primed. As predicted, the fragmenting identity leads to less socially efficient outcomes.

${ }^{5}$ Benjamin et al. (2010) try to assuage such fears by administering a questionnaire, asking subjects to guess the topic of the experiment. Although useful, this does not seem fully satisfactory, as the second set of questions could also be prone to experimenter demand effects and would only detect conscious biases.

${ }^{6}$ More generally, language has value as an alternative priming technology; it would be troubling if priming only worked if questionnaires were used.

${ }^{7}$ PGGs have been played extensively; for reviews see Zelmer's (2003) meta analysis, Cardenas and Carpenter's (2008) review of evidence from developing countries and Levitt and List's (2007) critique of the method. 
amongst one's own group. Second, a separate strand has compared contribution rates between different societies, since Roth et al.'s (1991) seminal work showed significant differences between subjects in four countries. More recent cross-country comparisons have shown that cross-society differences are correlated with characteristics such as their market integration or the strength of the rule of law, using comparisons between up to 16 societies (Henrich et al., 2001; Herrmann et al., 2008).

The are two fundamental differences between our paper and the PGG literature. First, the two strands of literature discussed above are interactions or comparisons between distinct societies or groups within a society. In our experiment, all subjects are sampled from the same single society, and all potentially belong to two groups: all have access to the same two distinct frames provided by their balanced bilingualism. The language of the experiment thus subtly makes salient one of two potential group memberships among all subjects in a session; this is new in the literature. We next investigate whether interactions in the group thus activated are affected by such priming. Our results provide support for the hypothesis that an individual's multiple group memberships (identities) have specific norms associated with them. ${ }^{8}$

Second, we study whether the effects of language on behaviour, through making a particular identity salient and thereby activating group-specific norms, are heterogeneous. Our subjects are all similar in one key respect and different in another. All subjects are accustomed to using either of the two languages for public communication. However for almost half of our subjects, one of the languages used, Lugisu, is also a language for the private sphere. The local (Gisu) culture is known in the ethnographic literature for valuing individual autonomy highly, whilst at the same time exhibiting outgroup favouritism (see Section 3.1). As mentioned, those most strongly associated with that culture contribute less in the local than in the national language, whereas for others this difference is not statistically significant. This result suggests that language activates group norms in a heterogeneous fashion: if different people's latent identities differ they can respond in very different ways to identical linguistic cues. We discuss this in the article borrowing insights from psycholinguistics and spell out implications for thinking about groups and public goods provision.

The article proceeds with Section 2 in which we describe the study area, sample, and implementation of the experiment. Section 3 derives our ethnographically motivated hypotheses in terms of a simple economic model of identity. Section 4 presents the main results, which are discussed in Section 5. Section 6 contains a brief conclusion.

\footnotetext{
${ }^{8}$ Akerlof and Kranton (2000, p.731) describe this case, where different identity-based pay-offs become salient in different situations, which may result from group-specific norms.
} 


\section{Experimental Details}

\subsection{Site and sample selection}

We selected as our study area the Nakaloke sub-county of Mbale in Eastern Uganda, a rural and predominately agricultural region with a population of 22,694 (Uganda Bureau of Statistics, 2014). We selected this sub-county because of its high level of balanced bilingualism. For historical reasons, there are two interchangeable languages for public purposes in Nakaloke: Luganda and Lugisu. ${ }^{9}$ Local informants estimate the degree of bilingualism of the two languages in question was $90 \%$ for language comprehension, but we used a strict screening process regarding language production which $68 \%$ met. ${ }^{10}$ It is locally expected that virtually all residents can communicate in both languages, but we wished to ensure subjects met a high bar of balanced fluency in the two languages. In the weeks before the experiment each potential subject was interviewed for some basic personal information, which gave an opportunity to assess whether they could fluently produce both languages.

Subjects were drawn from a multi-stage cluster randomisation such that they came from five randomly-selected villages in the area of four purposively-selected central meeting places (so twenty villages in total). In particular, we selected each of the four parishes of Nakaloke sub-county. These parishes consist of between 8 and 20 villages. We selected five villages randomly from each parish. For each village, we had a list drawn up of all $18+$ adults. From the list, we randomly selected 30 individuals (plus two spare) per village. 218 of the selected individuals were randomly assigned to the experiment reported on here, subject to the constraint that they were fluent in both Luganda and Lugisu. The remaining individuals were allocated to another experiment conducted in parallel. Central meeting places were chosen for ease of access and to mitigate the risk of cross-contamination between experimental days. Table 1 reports summary statistics for key characteristics for the sample as a whole and by treatment. ${ }^{11}$ This gives an insight

\footnotetext{
${ }^{9}$ The British colonisers collaborated with a Ganda general, Semei Kakungulu, to pacify the Gisu in the early 20th century. His followers from many different peoples settled in Nakaloke, part of the region of Bugisu, which therefore became ethnically highly diverse. The language spoken in Bugisu, Lugisu, was one natural lingua franca, and the language spoken among Kakungulu's followers, Luganda, became the other lingua franca (cf. Heald, 1998, pp. $23 \mathrm{ff}$. ).

${ }^{10}$ This was interpreted very strongly by local experimenters, so that $27.7 \%$ met only the Luganda standard, $3.1 \%$ met only the Lugisu standard and $1.3 \%$ met neither. In neighbouring sub-counties fluency in Luganda is less prevalent and fluency in Lugisu is higher. Another experiment was conducted simultaneously, and so subjects which did not meet the criterion of true bilingualism were selected, by default, into the other experiment.

${ }^{11}$ As shown in table 2 , the survey questions related to language and ethnicity were administered after the experiment. As such, we might expect that the answers on language use were influenced by the language of the experiment. The test statistics show no difference between treatments in the number of people reporting that Lugisu $(Z=1.07, p=0.28)$ or Luganda $(Z=1.24, p=0.22)$ is their main language at home. We wouldn't expect either of the two ethnic identity markers to be influenced by the language spoken, as they are less flexible.
} 
Table 1: Summary Statistics

\begin{tabular}{|c|c|c|c|c|}
\hline \multirow[b]{2}{*}{ Characteristic } & \multicolumn{2}{|c|}{ Whole Sample } & \multicolumn{2}{|c|}{ Frequency By Treatment } \\
\hline & Frequency & $\%$ of Sample & Luganda & Lugisu \\
\hline Total & 218 & 100 & 116 & 102 \\
\hline Male & 93 & 42.7 & 50 & 43 \\
\hline \multicolumn{5}{|c|}{ Education } \\
\hline None & 20 & 9.2 & 8 & 12 \\
\hline Some Primary & 102 & 46.8 & 57 & 45 \\
\hline Some Secondary & 90 & 41.3 & 47 & 43 \\
\hline Some Tertiary & 6 & 2.8 & 4 & 2 \\
\hline \multicolumn{5}{|c|}{ Religion } \\
\hline Muslim & 133 & 61.3 & 68 & 65 \\
\hline Catholic & 22 & 10.1 & 13 & 9 \\
\hline Born Again & 14 & 6.5 & 8 & 6 \\
\hline Anglican & 48 & 22.1 & 27 & 21 \\
\hline \multicolumn{5}{|c|}{ Tribe (inherited from Father) } \\
\hline Bagisu & 102 & 46.8 & 54 & 48 \\
\hline Baganda & 7 & 3.2 & 3 & 4 \\
\hline Bagwere & 43 & 19.7 & 21 & 22 \\
\hline Banyole & 33 & 15.1 & 19 & 14 \\
\hline Basoga & 18 & 8.3 & 10 & 8 \\
\hline Itesot & 6 & 2.8 & 4 & 2 \\
\hline Other & 9 & 4.1 & 5 & 4 \\
\hline \multicolumn{5}{|c|}{ Main Language Used at Home } \\
\hline Lugisu & 67 & 30.7 & 32 & 35 \\
\hline Luganda & 60 & 27.5 & 36 & 24 \\
\hline Lugwere & 65 & 29.8 & 33 & 32 \\
\hline Lunyole & 14 & 6.4 & 7 & 7 \\
\hline Lusoga & 8 & 3.7 & 5 & 3 \\
\hline Swahili & 1 & 0.5 & 0 & 1 \\
\hline English & 2 & 0.9 & 2 & 0 \\
\hline \multirow[t]{2}{*}{ Other } & 1 & 0.5 & 1 & 0 \\
\hline & Mean & $\mathrm{SD}$ & Mean & Mean \\
\hline Years in Nakaloke & 26.6 & 16.4 & 25.7 & 25.6 \\
\hline Age & 35.2 & 13.8 & 35.3 & 35.2 \\
\hline Acres of Land & 2.4 & 5.6 & 2.8 & 1.9 \\
\hline Number of Businesses & 0.83 & 0.97 & 0.9 & 0.8 \\
\hline
\end{tabular}

Other Linguistic and Ethnic Features

Number who use own tribe's language as main language at home $\quad 115$

Number whose father and mother come from the same tribe 115

Number whose spouse is from their tribe 59

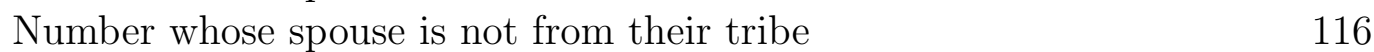

Perception of portion of the sub-county that shares the subject's own:

Very Few Few Some Most

\begin{tabular}{lrrrr} 
Main Language & 3 & 17 & 75 & 123 \\
Tribe & 4 & 14 & 104 & 96 \\
\hline
\end{tabular}


into the local language situation. For example, only 115 out of 218 subjects report using their tribe's language as their main language at home. Of the remaining 103, only 33 are using the language of their spouse's tribe. This explains how, while only $3.2 \%$ are Ganda, some $28 \%$ use Luganda (the associated language) as their main language at home. This language situation is not due to recent high levels of movement: only 21 subjects have lived in the region for fewer than 5 years, and 36 subjects for fewer than 10 years.

\subsection{Implementation of experiment}

We played a (slightly modified) one-shot linear public goods game. Sessions were conducted in either Lugisu or Luganda on four consecutive days in January 2013. All interactions between experimenters and subjects took place in a single language, from the elaborate, culturally prescribed welcoming act to unscripted directions regarding where to wait. Morning and afternoon sessions were run in each central location with a random order of the two languages. Communities are such that co-villagers would be expected to know each other, and other players would be a mixture of friends, acquaintances and unknowns. Each player was endowed with 8,000 Ugandan Shillings (UGS, a little over two daily wages for an agricultural labourer), in the form of twenty tokens each worth 400 UGS. Each subject was anonymously paired with another subject in the same session, which had between 18 and 30 subjects. Subjects chose to invest a number of tokens $g_{i}=[0, \ldots, 20]$ in the public good. The value of this public good was then increased by $50 \%$, and split equally between the two players. The payoff for each player is then calculated as $\pi_{i}=20-g_{i}+0.75\left(g_{i}+g_{j}\right)$ where $i$ is a given player, $j$ is their partner and the last term describes the payoff from the public good. The game is a social dilemma as the socially efficient choice is to contribute all of the endowment (such that each subject receives 12,000 UGS) but the dominant strategy is to contribute nothing.

We follow Charness and Dufwenberg (2010) in introducing a binary one-way cheap talk element, where each subject is randomly assigned to the role of either sender or receiver. The sender is able to send one of two messages to the receiver:

1. Let us both put everything into the box. That way we can both earn more money.

2. I won't put anything into the box. Let us each keep what we have.

The somewhat clunky English of these messages reflects the fairly literal back-translation from Luganda and Lugisu. The sender decides upon this message before deciding how much to contribute to the public good. While public goods games are typically taken as a measure of cooperation, the cheap talk design feature means that it is usefully extended to measure the extent of trusting/trustworthy behaviour, and includes some variation in expectations given the two signals. Both features of the data prove useful additions in discussing competing hypotheses. As we cannot guarantee literacy amongst our subject pool, 
each decision was communicated orally to one of two experimenters (see table 2 for details). The decision of which message to send was communicated to the first experimenter (who also asked three control questions), and the decision of how much to contribute to the public good was communicated to the second experimenter (who subsequently asked eight survey questions). The four combinations of contributing all/nothing are used as examples in the explanation, and control questions check understanding of the mechanisms. Of the 218 subjects 214 answered all three control questions correctly, and so we use all of the available data. ${ }^{12}$ The level of correct responses, and the triangulation of checking mechanisms while explaining corner solutions, give great confidence that subjects truly understood the game.

Table 2: Order

\begin{tabular}{lll}
\hline Sequence & Setting & Task \\
\hline 1 & Before Game Day & Assess language fluency \\
2 & In the Main Room & Experimental instructions \\
3 & With Experimenter 1 & Control questions, signal choice/reception \\
4 & With Experimenter 2 & Contribution choice, survey questions \\
\hline
\end{tabular}

A rigorous process of translation was used, where the local experimenter team (consisting of two experimenters involved in this experiment, and six in another) were involved in translating and/or checking the translation of the script to ensure comparability. The two experimenters used (who maintained their role in every session) are from the local area and live within the region, meaning they speak both languages with the local accent. Thankfully, neither of them was known in the sample villages.

\section{Gisu culture and hypotheses}

"Language is the repository of the history of a people."

Crystal (1997, p.20)

The violent history of the Gisu during the colonial era and its immediate aftermath shaped Gisu norms on cooperation in distinctive ways, which we expect to find their way into the Gisu's language, Lugisu. Combined with intense ecological pressure, this history gave rise to a marked absence of in-group loyalties, even to the point of out-group favouritism, as well as a high value placed on individual autonomy. In Section 3.1 we use the work of the Gisu's foremost recent ethnographer, Suzette Heald, to outline Gisu norms on cooperation and their origin, citing experimental evidence that supports her conclusions. In Section 3.2 we derive corresponding hypotheses, using a simple economic

\footnotetext{
${ }^{12}$ One subject did not answer questions relating to any land, business or religious affiliation, and so is excluded in related regressions.
} 
model of identity, about the effects of language use on willingness to contribute to a public good.

\subsection{Gisu norms on cooperation}

The first factor shaping Gisu norms on cooperation is ecological pressure. The Gisu are a people of smallholder farmers cultivating the slopes of Mount Elgon and its foothills in eastern Uganda, as well as the plains immediately to the west of these hills. They are about 1.3 million in number, almost all of them members of households economically dependent on their own land. Their agricultural plots are highly fertile, and population pressure on land in the region is intense, the current inhabitants being the descendants of the victors in a prolonged and violent struggle during the 19th century to secure its prized rich volcanic soils (Heald, 1998).

In a context of acute land shortage, the normal route to economic independence for a Gisu man is through early partible inheritance. After he is circumcised in late adolescence, which signifies his entry into manhood, a son may expect from his father a piece of land to start his own farm. A father thus divides land between his adult sons, keeping some for himself. The fairness of this division is scrutinised by relatives such a son's mother's brothers; and perceived unfairness in the distribution of land a frequent source of conflict, often violent, among kin (Hargreaves Heap et al., 2012; Heald, 1998).

Ecological pressure, giving rise to competition among kin over scarce resources, combines with the colonial legacy to explain the marked absence of in-group loyalties among the Gisu. The first three-quarters of the 20th century saw first subjugation, then oppression and finally anarchy for the Gisu. The British colonial administration imposed its 'rule by proxy' through the use of Ganda agents. In 1900, the British instructed a Ganda general, Semei Kakungulu, to subdue the Gisu and other peoples of what is now eastern Uganda. As mentioned earlier, Kakungulu settled in Nakaloke, the study area, together with his followers, which is the historical reason that levels of bilingualism are unusually high there.

Dating from the early days of the colonial era, the Gisu acquired a reputation for particularly violent and unruly behaviour (La Fontaine, 1959), a perception that may well have been an undeserved refraction through Ganda eyes (Heald, 1998, pp.22-30). It is true that they often rebelled against and sometimes murdered their Ganda overlords, set fire to their houses, and destroyed by night the work they had been forced to do by day as tribute labour; from the Gisu perspective, the Ganda were the oppressors. Such impertinence was usually met by collective punishment: punitive expeditions to kill, maim and imprison groups of Gisu associated with the wrongdoing, without an attempt to apportion individual blame, sufficiently harsh to act as a deterrent. The option of collective punishment was only rejected when "the District Officer [...] knew of no bond 
[...] which should lead them to incur punishment on each other's behalf" (Heald, 1998, p.155).

In the aftermath of Uganda obtaining independence from Britain in 1962, the situation in Bugisu approached anarchy. A power vacuum filled by psychotic rulers at the state level (Milton Obote, Idi Amin), the collapse of local governance structures and severe ecological pressure combined to produce chaos and uncertainty, in which petty theft and witchcraft accusations (mishaps were and are commonly attributed to supernatural malevolence) were rife. A common sight in Bugisu in the 1960s were expeditions of vigilantes (mirroring the Ganda expeditions to punish the Gisu during the colonial era) searching out alleged witches and thieves who, when found, would invariably be severely beaten and frequently killed. Strikingly, these killings often took place among kin and are a major contributing factor in Bugisu's high homicide rate: 32 per 100,000 during the mid 1960s, which was on a par with the highest recorded rates in the world at the time (Heald, 1998, p.31).

The lack of kinship loyalties, arising from the twin historical factors of competition within the lineage over scarce resources and the survival need to rapidly disown (deny a bond) with miscreant family members, is a remarkable feature of communal relations on a continent renowned for the strength of its kinship ties. Its corollary is an extraordinary value placed on individual autonomy. The Gisu are known for their emphasis on selfdetermination and self-reliance: values of autonomy are instilled in them from a young age. From early adulthood onwards, the Gisu are expected to make it through life depending on their own individual endeavours: neither expecting nor relying on assistance from others, nourishing and taking pride in the self-sufficiency of the individual. The necessity of cooperation and reliance on others (although real in practice) is systematically downplayed in the admonitions to the young. Suzette Heald puts it as follows.

"The idea of the individual, and individual autonomy [...] is strongly emphasised among the Gisu[:] the onus is upon the individual. Gisu individualism [...] systematically underplays the dependence of people upon each other. [Gisu society] sees itself as a society of self-determining autonomous individuals."

(Heald, 1998, pp.76-77)

These expectations of self-sufficiency and self-reliance apply to how the Gisu run their own affairs: in the "private sphere" an individual Gisu is expected to manage without needing to cooperate with others. Because they tend to live on their ancestral land, the Gisu live among their kin: their neighbours and co-villagers are frequently relatives. Their relations within the vicinity of where they live are thus characterised by a marked absence of the mutuality that one would expect to find in an African village. By contrast, in the "public sphere", these norms do not apply: their relations with outsiders are even marked by the reverse. The Gisu are highly unusual for exhibiting out-group favouritism: 
in experimental trust games, they send significantly more to those outside the village and lineage than those within (Hargreaves Heap et al., 2009, 2012). In the light of the Gisu's historical fears of outsiders (notably the Ganda during the colonial era), the authors interpret this as showing goodwill to prevent potential conflict from being actualised: preemptive appeasement, in other words.

It is of course historically plausible that the marked weakness of identification with the ingroup is simply the other side of the coin of the courting of favour with the outgroup: aggression from the latter could be avoided by a readiness to disown one's own.

\subsection{Hypotheses}

Building on Akerlof and Kranton's (2000) formal economic modelling of psychology's selfcategorisation theory, Benjamin et al. (2010) present a simple model of identity which provides a useful framework for analysing this experiment. They model a person as balancing what they would like to do in a given situation in the absence of any identity considerations with choice prescribed by any relevant category norms. Essentially a person's choice then becomes about weighing the disutility they feel from deviating from either of these two considerations. Priming experiments aim to affect the saliency of a category norm, and thus (partially) reveal it.

We aim to change the salience of different categories by changing the language of the experiment. The well-documented group norms for the Gisu people, which differ markedly from oft-proposed stylised facts for African village life are discussed at length in Section 3.1. To recap, both the weakness of identification with the ingroup and the default goodwill shown in relations with outsiders can be seen as arising from a violent past, in particular the indiscriminate, collective punishment meted out by the Ganda for Gisu disobedience, which could only be avoided by a readiness to dissociate oneself from fellow Gisu and appeasing the Ganda oppressors. The language of Luganda - especially in Nakaloke, the sub-county of Bugisu where Ganda general Semei Kakungulu settled with his followers in the early 20th century - would thus activate a contribution norm resulting in behaviour that clearly showed a keenness to cooperate. By contrast, the language of Lugisu would appeal to the values of autonomy that are instilled in the Gisu from an early age onwards.

We thus have the ethnographically rooted predictions that the contributions in the Luganda treatment will be higher than those in the Lugisu treatment. Note however that for the non-Gisu living in Gisu lands we have no ethnographic evidence that predicts the presence or absence of a treatment effect. The Gisu are the only people in the experiment who are appealed to in both a language that is associated with public norms (Luganda) and in a language that appeals specifically to their private norms (Lugisu). If 
the non-Gisu ${ }^{13}$ have internalised Gisu culture then the treatment effect should be found for all subjects, but the ethnographic literature is silent on this issue. We thus present two hypotheses: that the treatment effect will hold for all subjects (hypothesis 1a), and alternatively that it will only hold for those most associated with Gisu culture (hypothesis 1b). ${ }^{14}$

The null hypothesis of no treatment effect is to be expected if one holds to the 'fixedself' view of identity (see Hoff and Pandey, 2014, for discussion), where one's preferences are constant and not easily influenced in the short run. Alternatively, we would also expect the null hypothesis if language does not activate the different category norms.

Hypothesis 1a Average contributions will be higher in the Luganda treatment, for all subjects

Hypothesis 1b Average contributions will be higher in the Luganda treatment, but only for subjects that are most associated with Gisu culture

Null Hypothesis 1 Equal contributions in the two languages

Regarding the effects of 'bare promises' (where subjects can choose between a small number of messages), previous literature has tended to find small effects. In a trust game, Charness and Dufwenberg (2010) report binary cheap talk had a marginally significant trustworthiness-enhancing effect while the small trust-enhancing effect was not significant. These tests were low powered due to small samples, but those receiving or sending a blank message (as opposed to a 'cooperate' message) still cooperated in between a quarter and a half of cases. In a trust game with multiple rounds, Bracht and Feltovich (2009) compared the effects of controlled binary cheap talk and information on previous actions. Pooled tests for the average treatment effect of allowing cheap talk found no statistically significant difference, and they report only marginally significant effects on trusting behaviour in some rounds for receivers of the 'contribute' message. Thus we have null hypotheses 2 and 3, which expect no difference in trustworthy/trusting behaviour by signal.

Null Hypothesis 2 Equal contributions by signal sent

Null Hypothesis 3 Equal contributions by signal received 
Figure 1: Number of tokens contributed by Language and Game Role
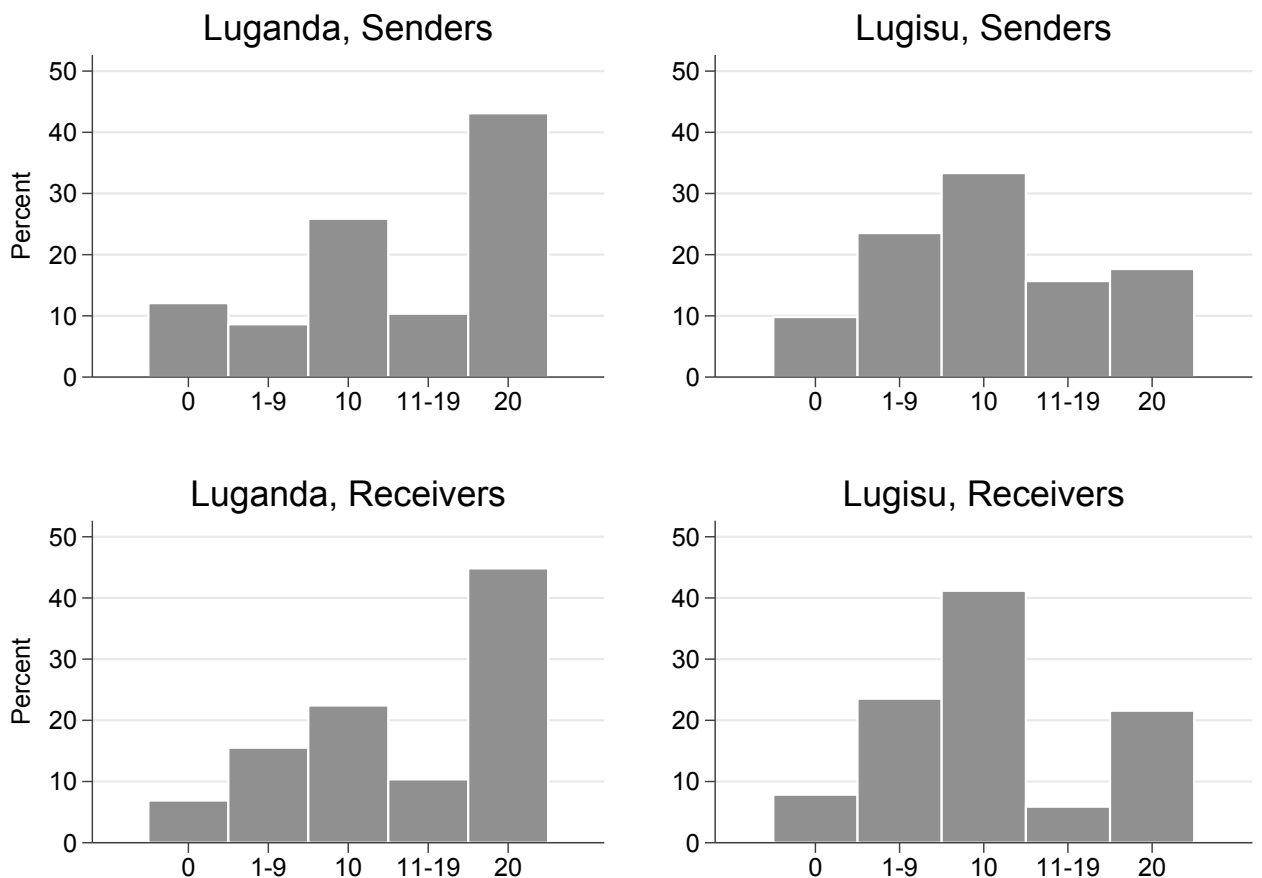

\section{Results}

Figure 1 shows the number of tokens contributed to the public good by language and game role. The number of tokens contributed are grouped into five categories: those contributing nothing, half, all or intermediate amounts (table 4 reports regressions using the same 5 point dependent variable). This is because these three focal points (contributing nothing, half or all) are the most popular options; there is discontinuity in the data.

Result 1 Contributions are significantly higher in the Luganda treatment than the Lugisu treatment.

On average subjects in the Lugisu treatment contribute $30 \%$ fewer tokens. The treatment effect is large (figure 1) and significant at all conventional levels (tables 3 and 4), and so null hypothesis 1 is rejected. This is also large compared to demographic correlates such as gender; on average men contribute $9.5 \%$ more than women. While a general shift to lower contributions is evident in figure 1, the striking difference is in the reduction of people who contribute all 20 tokens to the public good. For both senders and receivers,

\footnotetext{
${ }^{13}$ Table 1 provides information on the number of subjects from each tribe, where we follow the local custom of using the prefix ' $\mathrm{Ba}$ ' to denote tribe and ' $\mathrm{Lu}$ ' to denote language.

${ }^{14}$ We abstract from arguments to do with group size. Henrich $(2004$, p.11) argues that cooperation declines exponentially with group size, which implies that languages spoken by smaller numbers of people would, ceteris paribus, have higher cooperation (this result is contested within experimental economics, see Isaac et al., 1994).
} 
Table 3: Tests of Null Hypotheses

\begin{tabular}{lrrlrr}
\hline \multicolumn{7}{l}{ Null Hypothesis 1: Equal Contributions in the Two Languages } \\
Language & Luganda & Lugisu & Test & $\mathrm{T}$ Test & Mann-Whitney \\
Mean Contribution & 13.33 & 10.35 & Test Statistic & $\mathrm{T}=3.33$ & $\mathrm{Z}=3.35$ \\
$\mathrm{~N}$ & 116 & 102 & P Value & $\mathrm{P}<0.00$ & $\mathrm{P}<0.00$ \\
Null Hypothesis 2: Equal Contributions by Signal Sent & & \\
Signal & Contribute & Keep & Test & $\mathrm{T}$ Test & Mann-Whitney \\
Mean Contribution & 13.62 & 6.80 & Test Statistic & $\mathrm{T}=5.18$ & $\mathrm{Z}=4.44$ \\
$\mathrm{~N}$ & 79 & 30 & P Value & $\mathrm{P}<0.00$ & $\mathrm{P}<0.00$ \\
Null Hypothesis 3: Equal Contributions by & Signal Received \\
Signal & & \\
Mean Contribution & Contribute & Keep & Test & $\mathrm{T}$ Test & Mann-Whitney \\
N & 12.87 & 10.17 & Test Statistic & $\mathrm{T}=1.92$ & $\mathrm{Z}=1.99$ \\
& 79 & 30 & P Value & $\mathrm{P}=0.06$ & $\mathrm{P}=0.047$
\end{tabular}

Note: The dependent variable is the number of tokens contributed, out of a possible 20 . P values are two tailed.

\begin{tabular}{cll} 
Table 4: Treatment Effect, & \multicolumn{3}{c}{ Signal and } & Games Roles \\
\hline Controls & No & Yes \\
Variable & $(1)$ & $(2)$ \\
\hline Lugisu Session & $-0.585^{* * *}$ & $-0.595^{* * *}$ \\
& $(-3.21)$ & $(-3.56)$ \\
Sender * Don't Contribute & $-1.302^{* * *}$ & $-1.228^{* * *}$ \\
& $(-4.68)$ & $(-4.20)$ \\
Sender * Contribute & -0.152 & -0.0233 \\
& $(-1.02)$ & $(-0.22)$ \\
Receiver * Don't Contribute & $-0.735^{* *}$ & $-0.760^{* *}$ \\
& $(-2.10)$ & $(-1.99)$ \\
\hline $\mathrm{N}$ & 218 & 217 \\
$\mathrm{R}^{2}$ & 0.16 & 0.25 \\
\hline
\end{tabular}

Note: The dependent variable is the number of tokens contributed to the public good, but has been transformed to span 1-5, as in figure 1. Following Cameron et al. (2008) and Cameron and Miller (2015), we use the stata command cgmreg to calculate Wild cluster robust t-statistics at the session level, shown in parentheses. Controls are included but not reported. Personal characteristics controlled for are a subject's age, gender, time resident in the sub-county, education, wealth and religion.

Table 5: Tests Relating to Bagisu-Influenced Subjects

\begin{tabular}{lrrrrrr}
\hline Treatment: & \multicolumn{2}{c}{ Luganda } & \multicolumn{2}{c}{ Lugisu } & \multicolumn{3}{c}{ Tests } \\
Personal Characteristics & Mean & Obs & Mean & Obs & T Test & P Value \\
\hline Bagisu & 15.35 & 54 & 10.04 & 48 & 4.27 & $\mathrm{P}<0.000$ \\
Not Bagisu & 11.56 & 62 & 10.63 & 54 & 0.76 & $\mathrm{P}=0.449$ \\
Bagisu Mother & 14.34 & 58 & 10.00 & 55 & 3.53 & $\mathrm{P}<0.001$ \\
Non-Bagisu Mother & 12.31 & 58 & 10.76 & 47 & 1.20 & $\mathrm{P}=0.235$ \\
Main Language is Lugisu & 15.94 & 32 & 9.14 & 35 & 4.43 & $\mathrm{P}<0.000$ \\
Main Language is not Lugisu & 12.33 & 84 & 10.99 & 67 & 1.26 & $\mathrm{P}=0.211$ \\
\hline
\end{tabular}

Note: The dependent variable is the number of tokens contributed, out of a possible 20. P values are two tailed. 
just over $40 \%$ of subjects in the Luganda treatment contribute everything, while in the Lugisu treatment only around $20 \%$ of people do the same.

Result 2 Messages contain some value and are treated as such, but strategic play is limited.

Tables 3 and 4 show that both senders and receivers of signal 2 contribute fewer tokens to the public good, rejecting null hypotheses 2 and 3. For senders the difference is large (those sending signal 1 on average contribute twice as many tokens as senders of signal 2) and highly significant. Whilst always significant, the difference for receivers is much more modest: average contributions of receivers of signal 1 are only one quarter higher than those of signal 2. There are still considerable contributions even when subjects have received or given a signal that suggests their partner will not do the same: senders of signal 2 contribute around $37 \%$ on average, with receivers' contributing around $50 \%$.

The limited adjustment to the different signals is in line with the existing literature (discussed in Section 3.2), but it is still perhaps puzzling that the difference between signals is not larger, especially for receivers. ${ }^{15}$ Possible reasons fit into three main groups. First, it is possible that subjects didn't fully understand or trust these messages. We think it unlikely that comprehension was the driving factor (the excellent comprehension of the game is discussed in section 2.2, and the messages appear simpler than the game), but it is possible that because the messages weren't explained in the main room (see table 2) subjects didn't fully understand or trust the message sent/received. Second, it is possible that subjects did respond, but only partially. This could be because the message was binary (either contributing all or nothing) so didn't reflect a subject's true intentions, or simply because subjects only partially adjusted to the message having previously decided upon their preferred contribution level. Third, it could be the subjects were unconditional cooperators, or even gained greater utility in giving more than their partner. As such, they could reduce their partners' likely contribution, or ignore their claimed low contribution, and enjoy contributing more. These arguments lead to a different question: if the difference for receivers is justifiably small, what explains the much larger difference for senders? We would expect a larger difference for senders than receivers, as the difference for senders conflates the response to the signal with the straightforward effect of different types truthfully signalling their intention. ${ }^{16}$ We aren't able to distinguish between the

\footnotetext{
${ }^{15}$ While the average difference in contributions in response to the two messages is relatively limited, the difference in the distributions is more marked. For receivers of signal 1, the percentages of those giving under, exactly or over half of the tokens are (rounding) $22 \%, 32 \%$ and $47 \%$, whereas for those receiving signal 2 those numbers are respectively $40 \%, 30 \%$ and $30 \%$.

${ }^{16}$ The frequency of the "let's pool" message is virtually identical in the Luganda and Lugisu treatment: $72.4 \%$ and $72.5 \%$, respectively. Both senders $(t=2.82 ; p=0.005)$ and receivers $(t=1.93 ; p=0.055)$ contribute more in Luganda than in Lugisu when the message is "let's pool". Conditional on the "let's keep" message, receivers of the message in Luganda contribute more than those in Lugisu $(t=1.78 ; p=$ $0.077)$, as do senders, although in this case the difference is not significant $(t=0.56 ; p=0.576)$. All of these tests are two-sided.
} 
relative strength, if any, of these different factors but suspect a combination limits the strength of any response to bare promises.

Table 6: Language Effect, by Identity Sub-Group

\begin{tabular}{lrrrrrr}
\hline Gisu ID Marker: & \multicolumn{2}{c}{ Gisu (Father) } & \multicolumn{2}{c}{ Gisu Mother } & \multicolumn{2}{c}{ Lugisu Speaker } \\
Controls & No & Yes & No & Yes & No & Yes \\
Variable & $(1)$ & $(2)$ & $(3)$ & $(4)$ & $(5)$ & $(6)$ \\
\hline Luganda * Gisu ID & $0.878^{* * *}$ & $0.862^{* * *}$ & $0.546^{* * *}$ & $0.540^{* * *}$ & $0.899^{* * *}$ & $0.742^{* * *}$ \\
& $(6.92)$ & $(10.00)$ & $(3.87)$ & $(4.91)$ & $(6.73)$ & $(4.87)$ \\
Lugisu * Gisu ID & -0.233 & -0.272 & -0.315 & -0.329 & $-0.522^{* *}$ & $-0.678^{* * *}$ \\
& $(-1.12)$ & $(-1.07)$ & $(-1.45)$ & $(-1.46)$ & $(-2.50)$ & $(-2.64)$ \\
\hline Observations & 218 & 217 & 218 & 217 & 218 & 217 \\
$\mathrm{R}^{2}$ & 0.10 & 0.19 & 0.06 & 0.15 & 0.09 & 0.18 \\
\hline
\end{tabular}

Note: The dependent variable is the number of tokens contributed to the public good, but has been transformed to span 1-5, as in figure 1. Wild cluster robust t-statistics are shown in parentheses. A constant is included but not reported.

Result 3 The treatment difference is due to those most associated with Gisu culture.

Tables 5 and 6 present the related evidence for our experimental results, showing support for hypothesis 1 b over 1a. Specifically, we should expect to see lower contributions, due to the norm of autonomy, for people who have internalised ${ }^{17}$ (to some extent) Gisu culture when Gisu culture is primed. The tables present three different ways of separating the data relating to Gisu identity: father is (not) Gisu, mother is (not) Gisu and main language at home is (not) Lugisu. Only the first of these comparisons is strictly ethnic, as in Ugandan culture ethnicity is a paternal inheritance. Table 5 shows that in each of the three ways of separating the data, there is only evidence of a treatment effect (average contributions are higher by 43-74\%) where subjects may be expected to have internalised Gisu culture and norms. In no case in table 5 is there a treatment effect for non-Gisu-influenced subjects. Table 6 tests only for treatment effects for Gisu-influenced subjects, using the same three identity markers. In each case, Gisu-influenced subjects give significantly more in Luganda (at the $1 \%$ level in every specification) and less in Lugisu (with various significance levels in different specifications) than non-Gisu-influenced subjects. The largest treatment effects are found when using language itself as the identity marker. ${ }^{18}$

\footnotetext{
${ }^{17}$ There is no clear and precise theory of what constitutes being 'bicultural', nor an accepted way of measuring it. We use the terms 'internalised Gisu identity' and 'Gisu-influenced subjects' as shorthand to refer to subjects that are captured using the three markers we can measure the concept with: ethnicity, mother's ethnicity and language spoken at home.

${ }^{18}$ Using the data we collect on what a subject gives as their main language in general (rather than at home), we can rule out comprehension as a confounding factor. Restricting the sample to subjects that meet a Gisu identity marker and give Luganda as their main language in general, we find significant
} 
Result 4 The difference in behaviour for those most associated with Gisu culture appears to be due to unconditional (non) cooperation.

There are two main mechanisms that could be underpinning Result 3, as the greater cooperation of Gisu-influenced subjects in Luganda could be either conditional or unconditional. It would be conditional cooperation if Gisu subjects had different expectations over the partner's identity or expected their partner to behave in a different way. The former channel does not seem plausible, as the script emphasises that one's partner has been randomly sampled from the Nakaloke subcounty, which has a population of only 22,694 (Uganda Bureau of Statistics, 2014). Local informants reported that both languages were widely understood and used, and so neither language implies excluding a portion of that sub-county. There may still be conditional cooperation if subjects' expectations of their partner's behaviour differs by language. The cheap talk design element is useful here, as it provides strong differences in expectations. ${ }^{19}$ As shown in Result 2 subjects contribute more on average when the contribute message is chosen, showing that cooperation amongst our subject pool as a whole does have a significant conditional aspect.

Table 7: Tests of Conditional Cooperation, Receivers

\begin{tabular}{|c|c|c|c|}
\hline Gisu ID Marker: & $\begin{array}{r}\text { Gisu (Father) } \\
(1)\end{array}$ & $\begin{array}{r}\text { Gisu Mother } \\
(2)\end{array}$ & $\begin{array}{r}\text { Lugisu Speaker } \\
\text { (3) }\end{array}$ \\
\hline \multirow[t]{2}{*}{ Non-Gisu*Cooperate } & $0.812^{* *}$ & 0.195 & -0.150 \\
\hline & $(2.15)$ & $(0.82)$ & $(-0.48)$ \\
\hline \multirow[t]{2}{*}{ Gisu*Cooperate } & $1.100^{* *}$ & 0.377 & $-0.674^{* *}$ \\
\hline & $(2.29)$ & $(0.90)$ & $(-2.39)$ \\
\hline \multirow[t]{2}{*}{ Gisu*Don't Cooperate } & $0.670 * * *$ & 0.329 & -0.203 \\
\hline & $(2.61)$ & $(0.60)$ & $(-0.81)$ \\
\hline \multirow[t]{2}{*}{ Constant } & $2.643^{* * *}$ & $3.217^{* * *}$ & $3.674^{* * *}$ \\
\hline & $(9.15)$ & (17.67) & (13.44) \\
\hline Observations & 109 & 109 & 109 \\
\hline $\mathrm{R}^{2}$ & 0.06 & 0.01 & 0.03 \\
\hline
\end{tabular}

Note: The dependent variable is the number of tokens contributed to the public good, but has been transformed to span 1-5, as in figure 1. Wild cluster robust t-statistics are shown in parentheses. The excluded category is non-Gisu receiving the 'don't cooperate' message, and all other coefficients should be interpreted in conjunction with the constant term. Senders of a message are excluded, in order to have a cleaner test.

Basic statistics in table 3 show that subjects that receive the contribute message gave an average of $27 \%$ more than those receiving the don't contribute message, but this hides

treatment effects for Gisu subjects $\left(t=2.30^{* *}, N=28\right)$, subjects with a Gisu mother $\left(t=1.78^{*}, N=40\right)$ and subjects whose main language at home is Lugisu $\left(t=5.87^{* * *}, N=19\right)$. All tests are two-tailed. Our thanks to a referee for pointing us to this test.

${ }^{19}$ Whilst possibly desirable, neither the strategy method nor the elicitation of expectations were likely to be feasible amongst our subject pool without a fall in comprehension. 
heterogeneity. Conditional cooperation characterises non Gisu-influenced subjects, who contribute significantly more on average when receiving the cooperate message (42-46\%, depending on the identity marker used). However, for Gisu-influenced subjects there is little difference (on average, contributions are only higher by 4-19\%). Table 7 shows regression results for the effect of the signal on receivers interacted with Gisu-influenced identity markers. We can see in column one that non-Gisu receiving the cooperate message give significantly more on average than non-Gisu receiving the other message (the excluded category), which is significant at the $5 \%$ level. (This difference is not significant in any other column, using different identity markers.) While the non-Gisu can be interpreted directly, for the Gisu a test of the equality of coefficients is needed. For Gisu (chi=1.13, p=0.29), Gisu-mother (chi=0.01, p=0.93) and Lugisu speaking (chi=1.34, $\mathrm{p}=0.25$ ) identity markers, there is never a significant difference by the signal received. In other words, there is no evidence for conditional cooperation for Gisu-influenced subjects, only for non-Gisu-influenced subjects. These results provide some evidence that the mechanism for greater contributions by Gisu-influenced subjects is unconditional cooperation in Luganda and unconditional non-cooperation in Lugisu. In other words, their absence of a response to the message suggests that language affects not what they think their partner will do but what they think they themselves should do. The statistical power is not sufficient to look much more closely at interaction effects or sub-groups but the pattern is consistent across the three identity markers, providing suggestive evidence of the underlying mechanism.

\section{Discussion}

We study the importance of language in making salient one of potentially multiple identities. As indicated in the introduction, the study of language is limited among economists, and its connection with identity even more so. Exceptions include two studies that use survey data to examine whether the introduction of bilingual education strengthens the identity associated with the newly introduced language of instruction (Aspachs-Bracons et al., 2008; Clots-Figueras and Masella, 2013). The use of survey data leaves unexamined the role of a potentially large number of confounds. Amongst the limited number of experimental approaches, Li (2010) measures the preferences of students in Hong Kong using English and Chinese. Subjects are found to be more prosocial in Chinese in trust games, with no difference in (various) dictator games. It is unclear whether the lack of significance is due to low-powered $\operatorname{tests}^{20}$ (the total sample size is 63 subjects) or because Hong Kong students only really have one identity (and so languages can't activate different sets of norms).

\footnotetext{
${ }^{20}$ Another potential confound are order effects: subjects make a minimum of 31 decisions in around 50 minutes, and the only significant differences are found for games 7, 8 and 9 out of the 10 played.
} 
There are two experimental papers where subjects are expected to have different identities, and where these are overtly primed using different languages. In a field experiment Lambarraa and Riener (2015) use treatments in Arabic and French to examine donations to charity among Moroccan participants. The aim is to find out whether anonymity increases giving, as Islam encourages giving in secret. The purpose of using Arabic is to remind subjects of their religious duties in Islam, in particular anonymous gifts to good causes. A religious identity is thus primed, but the use of language is incidental: a treatment in French that states two relevant verses from the Qur'an leads to even more giving. In a related paper Espinosa et al. (2015) use Basque/Spanish and Catalan/Spanish treatments in two places in Spain, with local (club) and global public goods. Subjects choose which language they prefer to play in, and are randomised between homogeneous treatments (where adjoining rooms play in the same language) and heterogeneous treatments (where different rooms play in different languages). The language used in the other room is announced, and so (as in Lambarraa and Riener, 2015) the use of language is in a sense exploiting experimenter demand effects to activate associated norms. They find that in the Basque country (where there is a salient identity conflict) heterogeneous treatments saw contributions to the global public good fall, whereas in Valencia county (where identity conflict is not a live issue) there is no significant difference. Our experiment differs from these two papers as we do not point subjects strongly in a particular direction, our concern is rather with using language to innocuously prime subjects in order to reveal a social identity that remains latent when that language is not used.

\subsection{Language and identity are connected}

With multilingualism being the global norm, the study of language and identity clearly has a large reach. We selected a bilingual subject pool who, for historical reasons, have two de facto lingua francas. One of these, Lugisu, is associated with a well-documented social identity of remarkable individual autonomy (by African standards), combined with outgroup favouritism. As both languages are public languages and can be used interchangeably in the study area, the use of one or the other as the language of instruction in the experiments provides a seemingly innocuous cue as to the social identity that is being appealed to. We therefore present experimentally-obtained evidence on the connection of language and identity for which language has been used as a subtle priming technology, untroubled by experimenter demand effects. Moreover, we take advantage of heterogeneity in the subject pool. For a considerable proportion of our sample, one of the lingua francas is also a private language, whereas for the remainder both are only for public use.

We find that contributions to the public good are much higher in the Luganda treatment and that this is driven by the behaviour of those most strongly associated with the Gisu culture. In psychology, it would be natural to interpret this finding in terms of a the- 
ory of bilingualism called dynamic constructivism. Mirroring Akerlof and Kranton (2000), Hong et al.'s (2000) work was seminal in psychology as it presented empirical evidence in support of the dynamic constructivist approach that argues biculturals have a variety of cultural frames which when primed become dominant. Luna et al. (2008) extended this work by using languages as their priming technology, showing in their experiments (using non-incentivised survey questions and small samples) that only bicultural subjects were influenced by the language prime. They argue that the different cultural frames only exist for people who have internalised two cultures, and only for them will priming lead to different cultural frames of reference. In our experiment, the Gisu are biculturals: for them Luganda is a language for the public sphere and Lugisu (also) a language for the private sphere. For everybody else, both languages are only for the public sphere; their position in our experiment is that of monoculturals. ${ }^{21}$ The crucial distinction between biculturals and monoculturals in our experiment is that the former's various identities are appealed to once through a language that belongs only to the public sphere and once through a language that belongs also to the private sphere; the latter are only ever instructed in languages for public use. Dynamic constructivism predicts that language is a cue for identity for biculturals but not for monoculturals, which is consistent with what we find.

\subsection{Moving beyond mutually exclusive groups}

As well as providing experimental evidence on a link between language and identity, we contribute a nuance to the experimental literature on cultural differences in willingness to contribute to a public good. ${ }^{22}$ PGGs that are conducted to explore cultural differences between different groups can be interpreted, in the language of Benjamin et al.'s (2010) model, as examining differences in behaviour assuming no category norms have been made salient. Previous research is thus looking for static differences between societies, whereas our paper examines how multiple group identities, triggered by language, affect economic behaviour. It is an attempt to reveal behaviour mediated through category norms; and to examine whether multiple category norms may co-exist. ${ }^{23}$ In doing so, we study both the way in which different group norms can be activated and how these group norms have heterogeneous effects on category members.

We believe this is a more useful and realistic account of human behaviour. People rarely have one identity or group membership, with a clearly defined in- and out-group.

\footnotetext{
${ }^{21}$ Strictly speaking, the $7(3.2 \%)$ Baganda are also biculturals in our experiment; we do not have clear predictions regarding these, nor the statistical power to test any.

${ }^{22}$ For a brief review of this literature, see the introduction.

${ }^{23}$ As Benjamin et al. (2013, p.16) argue, priming only reveals behaviour that is mediated through category norms, not behaviour that is mediated through what a person would like to do in the absence of identity considerations. As such, subjects who have fully internalised the norms of a particular category will not be affected by priming. In the context of our experiment, subjects who truly hold autonomy as an ideal will not be influenced by the Lugisu prime but contribute no tokens in all settings.
} 
To take a commonplace example, the authors of this article share a common European identity but not a national one. Outside of the lab and well-publicised episodes of ethnic violence, ethnic identities are rarely clear-cut or mutually exclusive. As such, the results presented here are informative. Our experimental subjects are all members of at least two language groups, but these memberships trigger different norms depending on one's exposure to group norms. Different groups have different norms, and daily life for the majority of the world's population involves interacting with people who in one context are group members and in other contexts are not. Contextual cues may activate group-specific norms and we have shown that in a multilingual setting one such cue is language.

\subsection{A common language may encourage cooperation}

Finally, our findings suggest an optimistic twist to the common finding that ethnic diversity leads to lower provision of public goods (Alesina et al., 1999; Miguel and Gugerty, 2005 , etc.). Take a group such as the Gisu, who value individual autonomy when left to themselves. By contrast, when members of this group find themselves in an ethnically diverse society, they may become more willing to contribute to public goods than they would have been in an unfragmented Gisu society. The trigger for such public-spirited norms is sharing a common language with other groups in a society. The father of modern linguistics, de Saussure (1959, p.14) argued that language itself is an example of cooperation as it exists "only by virtue of a sort of contract signed by members of a community". This provides the hope that more ethnically diverse societies are not condemned to higher crime, worse schools, dirtier drinking water, and so forth. A common language may activate norms of cooperation. This chimes with the findings and references in Glennerster et al. (2013), where Sierra Leone's common language is used to explain why its high ethnic diversity has not undermined the provision of public goods.

\section{Conclusion}

We present the results from a Public Goods Game amongst a bilingual population in Eastern Uganda. Subjects are randomly assigned to a Luganda treatment or a Lugisu treatment. For historical reasons, these two languages are interchangeable for public purposes in the study area. We test the hypothesis that the two languages will invoke different aspects of the social identity associated with the local Gisu culture, which highly values both showing goodwill in dealings with outsiders and individual autonomy in dayto-day living. We find strong support for this hypothesis: on average contributions are some $30 \%$ higher in the Luganda treatment than in the Lugisu treatment. Furthermore, this effect is entirely driven by the behaviour of those most closely associated with the local Gisu culture (which is not the same as being Gisu). 
The findings suggest that language and identity are intimately connected. Groupspecific norms associated with a particular identity may be activated by the language that acts as a cue for that identity and remain latent when another language is used. In addition, it seems that people who share an identity may not place the same weight on it when responding to identical contextual cues. With multilingualism being the global norm, we take from our findings an important implication for the prospects for cooperation in multilingual societies. Groups are neither static nor mutually exclusive; in an important sense, groups only come into being in response to particular contextual cues. We have shown that one such cue is language. Group-specific norms vary in terms of the cooperativeness they prescribe and may be activated or remain latent. A common language connected with a social identity of being cooperative may thus encourage cooperation, but only for people who have internalised the norms of that identity. 


\section{References}

Akerlof, G. A. and Kranton, R. E. (2000). Economics and identity. The Quarterly Journal of Economics, 115(3):715-753.

Alesina, A., Baqir, R., and Easterly, W. (1999). Public goods and ethnic divisions. The Quarterly Journal of Economics, 114(November):pp.1243-1284.

Aspachs-Bracons, O., Clots-Figueras, I., Costa-Font, J., and Masella, P. (2008). Compulsory language educational policies and identity formation. Journal of the European Economic Association, 6(2-3):pp.434-444.

Banerjee, A., Iyer, L., and Somanathan, R. (2005). History, social divisions, and public goods in rural india. Journal of the European Economic Association, 3(2-3):639-647.

Benjamin, D., Choi, J., and Fisher, G. (2013). Religious Identity and Economic Behaviour. Working Paper.

Benjamin, D. J., Choi, J. J., and Strickland, A. J. (2010). Social identity and preferences. American Economic Review, 100:1913-1928.

Bernhard, H., Fehr, E., and Fischbacher, U. (2006). Group affiliation and altruistic norm enforcement. The American Economic Review, 96(2):217-221.

Bracht, J. and Feltovich, N. (2009). Whatever you say, your reputation precedes you: Observation and cheap talk in the trust game. Journal of Public Economics, 93(9):10361044.

Cadsby, C. B., Servátka, M., and Song, F. (2013). How competitive are female professionals? A tale of identity conflict. Journal of Economic Behavior \& Organization, 92:284-303.

Cameron, A. C., Gelbach, J. B., and Miller, D. L. (2008). Bootstrap-based improvements for inference with clustered errors. The Review of Economics and Statistics, 90(3):414427.

Cameron, A. C. and Miller, D. L. (2015). A practitioner's guide to cluster-robust inference. Journal of Human Resources, 50(2):317-372.

Cardenas, J. C. and Carpenter, J. (2008). Behavioural development economics: lessons from field labs in the developing world. The Journal of Development Studies, 44(3):311338.

Charness, G. and Dufwenberg, M. (2010). Bare promises: An experiment. Economics Letters, 107(2):281-283.

Chen, M. K. (2013). The Effect of Language on Economic Behavior: Evidence from Savings Rates, Health Behaviors, and Retirement Assets. American Economic Review, 103(2):690-731.

Chen, Y., Li, S. X., Liu, T. X., and Shih, M. (2014). Which hat to wear? Impact of natural identities on coordination and cooperation. Games and Economic Behavior, 84:58-86. 
Clots-Figueras, I. and Masella, P. (2013). Education, language and identity. The Economic Journal, 123(570):pp. 332-357.

Costa, A., Foucart, A., Arnon, I., Aparici, M., and Apesteguia, J. (2014a). "Piensa" twice: On the foreign language effect in decision making. Cognition, 130(2):236-254.

Costa, A., Foucart, A., Hayakawa, S., Aparici, M., Apesteguia, J., Heafner, J., and Keysar, B. (2014b). Your Morals Depend on Language. PloS one, 9(4):e94842.

Crystal, D. (1997). English as a Global Language. Cambridge, UK: Cambridge University Press.

de Saussure, F. (1959). Course in general linguistics. . New York: The Philosophical Library.

Desmet, K., Ortuño-Ortín, I., and Wacziarg, R. (2012). The political economy of linguistic cleavages. Journal of development Economics, 97(2):322-338.

Espinosa, M. P., Fatas, E., and Ubeda, P. (2015). Identity, language, and conflict: An experiment on ethno-linguistic diversity and group discrimination in two bilingual societies. CBESS Discussion Paper, 15(14).

Etang, A., Fielding, D., and Knowles, S. (2011). Does trust extend beyond the village? Experimental trust and social distance in Cameroon. Experimental Economics, 14(1):15-35.

Finocchiaro Castro, M. (2008). Where are you from? Cultural differences in public good experiments. The Journal of Socio-Economics, 37(6):2319-2329.

Glennerster, R., Miguel, E., and Rothenberg, A. D. (2013). Collective action in diverse sierra leone communities. The Economic Journal, 123(568):285-316.

Habyarimana, J., Humphreys, M., Posner, D. N., and Weinstein, J. M. (2009). Coethnicity: Diversity and the Dilemmas of Collective Action. Russell Sage Foundation: New York.

Hargreaves Heap, S., Verschoor, A., and Zizzo, D. J. (2009). Out-Group favouritism. Social Science Research Network, Available at SSRN: http://ssrn.com/abstract $=1428937$ or http://dx.doi.org/10.2139/ssrn.1428937.

Hargreaves Heap, S., Verschoor, A., and Zizzo, D. J. (2012). A test of the experimental method in the spirit of Popper. Journal of Economic Methodology, 19(1):63-76.

Heald, S. (1998). Controlling Anger: The anthropology of Gisu violence. Manchester, England: James Currey, Oxford.

Henrich, J. (2004). Cultural group selection, coevolutionary processes and large-scale cooperation. Journal of Economic Behavior \&3 Organization, 53(1):3-35.

Henrich, J., Boyd, R., Bowles, S., Camerer, C., Fehr, E., Gintis, H., and McElreath, R. (2001). In search of homo economicus: Behavioral experiments in 15 small-scale societies. The American Economic Review, Papers and Proceedings, 91(2):73-78.

Herrmann, B., Thöni, C., and Gächter, S. (2008). Antisocial Punishment Across Societies. Science, 319(5868):1362-1367. 
Hoff, K. and Pandey, P. (2006). Discrimination, social identity, and durable inequalities. The American Economic Review, 96(2):206-211.

Hoff, K. and Pandey, P. (2014). Making up people-The effect of identity on performance in a modernizing society. Journal of Development Economics, 106:118-131.

Hoff, K. and Stiglitz, J. E. (2016). Striving for balance in economics: Towards a theory of the social determination of behavior. Journal of Economic Behavior \& Organization, $126: 25-57$.

Hong, Y., Morris, M., Chiu, C., and Benet-Martinez, V. (2000). Multicultural minds: A dynamic constructivist approach to culture and cognition. American Psychologist, 55 (7):pp.709-720.

Isaac, R. M., Walker, J. M., and Williams, A. W. (1994). Group size and the voluntary provision of public goods: experimental evidence utilizing large groups. Journal of Public Economics, 54(1):1-36.

Keysar, B., Hayakawa, S. L., and An, S. G. (2012). The Foreign-Language Effect Thinking in a Foreign Tongue Reduces Decision Biases. Psychological science, 23(6):pp.661-668.

La Fontaine, J. S. (1959). The Gisu of Uganda. London, UK: International African Institute.

Lambarraa, F. and Riener, G. (2015). On the norms of charitable giving in Islam: Two field experiments in Morocco. Journal of Economic Behavior and Organization, (forthcoming).

LeBoeuf, R., Shafir, E., and Bayuk, J. (2010). The conflicting choices of alternating selves. Organizational Behavior and Human Decision Processes, 111(1):48-61.

Levitt, S. D. and List, J. A. (2007). What do laboratory experiments measuring social preferences reveal about the real world? The Journal of Economic Perspectives, pages $153-174$.

Lewis, M. P., Simons, G. F., and Fennig, C. D. (2013). Ethnologue: Languages of the World, Seventeenth edition. Dallas, Texas: SIL International.

Li, K. K. (2010). Thinking in chinese vs. thinking in english : Social preference and risk attitudes of multicultural minds. Jena economic research papers, 2010,061.

Luna, D., Ringberg, T., and Peracchio, L. A. (2008). One individual, two identities: Frame switching among biculturals. Journal of Consumer Research, 35(2):279-293.

Miguel, E. and Gugerty, M. K. (2005). Ethnic diversity, social sanctions, and public goods in Kenya. Journal of Public Economics, 89(December):2325-2368.

Romaine, S. (2001). The handbook of linguistics, chapter Multilingualism, pages 512-532. Blackwell: Oxford.

Roth, A. E., Prasnikar, V., Okuno-Fujiwara, M., and Zamir, S. (1991). Bargaining and market behavior in Jerusalem, Ljubljana, Pittsburgh, and Tokyo: An experimental study. The American Economic Review, 81(5):1068-1095.

Uganda Bureau of Statistics (2014). National Population and Housing Census 2014, 
Provisional Results. Kampala, Uganda: Uganda Bureau of Statistics.

Zelmer, J. (2003). Linear public goods experiments: A meta-analysis. Experimental Economics, 6(3):pp.299-310.

Zizzo, D. J. (2010). Experimenter demand effects in economic experiments. Experimental Economics, 13(1):pp.75-98. 